\title{
Acesso aos serviços de atenção primária à saúde no Sistema Único de Saúde: Fortalezas e desafios emergentes das experiencias apresentadas ao Prêmio "APS Forte para o SUS"
}

\author{
Renato Tasca, Wellington Carvalho, Rosane Gomes, lasmine Ventura, Vanessa Pinheiro \\ Borges, Fernando Leles, Flávio Goulart e Julio Jimenez
}

\section{RESUMO}

O artigo visa identificar, dar visibilidade, reconhecer e promover iniciativas locais, municipais ou regionais que tenham como foco a melhoria do acesso aos serviços de APS. Esta iniciativa buscou experiências que promoveram este atributo, reforçando o papel da APS como porta de entrada prioritária e coordenadora da atenção no sistema de saúde.

Palavras-chave: Atenção Primária à Saúde; Acesso; Prêmio APS.

\section{ABSTRACT}

The article aims to identify, give visibility, recognize and promote local, municipal or regional initiatives that focus on improving access to PHC services. This initiative sought experiences that promoted this attribute, reinforcing the role of PHC as a priority gateway and coordinator of care in the health system.

Revista da Rede APS 2020

Publicada em: 04/09/2020

DOI:10.14295/aps.v2i3.145

Renato Tasca

(Organização Pan-Americana de Saúde)

Wellington Carvalho

(Organização Pan-Americana de Saúde)

Rosane Gomes

(Organização Pan-Americana de Saúde)

lasmine Ventura

(Organização Pan-Americana de Saúde)

Keywords: Prmary Health Care; Access; PHC Prize.

Vanessa Pinheiro Borges
(Organização Pan-Americana de
Saúde)
Fernando Leles
(Organização Pan-Americana de
Saúde)
Flávio Goulart
(Organização Pan-Americana de
Saúde)
Julio Jimenez
(Organização Pan-Americana de
Saúde)
Correspondência para:
Renato Tasca
renato.tasca@gmail.com




\section{Objetivos e metodologia de AVALIAÇÃO do PrÊMIO}

Em cinco de abril de 2019, no ano do 25은 aniversário da Estratégia Saúde da Família, a Organização Pan-americana de Saúde / Organização Mundial de Saúde (OPAS/OMS), em parceria com o Ministério da Saúde, lançou o Prêmio APS Forte para o SUS: Acesso Universal. O objetivo foi identificar, dar visibilidade, reconhecer e promover iniciativas locais, municipais ou regionais que tenham como foco a melhoria do acesso aos serviços de APS. A escolha da temática se deveu a um consenso das instituições patrocinadoras do prêmio de que o acesso é o atributo da Atenção Primária à Saúde (APS) que mais precisa ser fortalecido no Sistema Único de Saúde (SUS). Esta iniciativa buscou experiências que promoveram este atributo, reforçando o papel da APS como porta de entrada prioritária e coordenadora da atenção no sistema de saúde.

Durante os dois meses de inscrição (entre 15 de abril e 15 de junho de 2019), 1.294 experiências foram inscritas, de todos os estados do país. Para analisá-las, foi necessário um processo de sistematização dos relatos e apoio de diversas instituições colaboradoras. Esse processo ocorreu em 4 fases, que foram apresentadas com transparência aos participantes por meio da página virtual do Portal da Inovação na Gestão do SUS (www.apsredes.org):

Fase 1 - Admissibilidade: o Comitê organizador do Prêmio realizou um primeiro filtro das experiências, verificando aspectos formais das inscrições, eliminando aquelas que não respeitaram ao determinado no edital do Prêmio. Neste processo, 55 experiências foram eliminadas. Sendo assim, a partir desse momento, o número de experiências analisadas passou a ser de 1.239. A partir de então, foi realizada a categorização em blocos, considerando categorias conceituais, para distribuição aos avaliadores.

Fase 2 - Análise e classificação das experiências: nessa fase, se conformou o Grupo de Avaliadores do Prêmio, composto por técnicos indicados pelas seguintes instituições:
Associação Brasileira de Enfermagem de Família e Comunidade - ABEFACO; Associação Brasileira de Saúde Coletiva - ABRASCO; Conselho Nacional de Saúde - CNS; Conselho Nacional das Secretarias Municipais de Saúde - CONASEMS; Conselho Nacional das Secretários de Saúde CONASS; Ministério da Saúde - MS; Organização Pan-americana da Saúde / Organização Mundial da - OPAS/OMS; Saúde Sociedade Brasileira de Medicina de Família e Comunidade - SBMFC. Cada experiência foi avaliada por dois avaliadores e ao final desse processo, obteve-se 135 experiências classificadas para a próxima fase. Mais de cem profissionais, de diversas disciplinas, colaboraram para realizar este processo, utilizando instrumentos compartilhados.

Fase 3 - Classificação dos Finalistas: as 135 experiências classificadas como "recomendadas para o prêmio", na fase anterior, foram avaliadas pelo Comitê do Prêmio, composto pela OPAS/OMS, MS, CONASS, CONASEMS e CNS. Após análise individual das experiências por cada instituição e 3 reuniões de consenso do Comitê, definiu-se as 11 finalistas do Prêmio.

Fase 4 - Seleção dos 3 premiados: O Comitê do Prêmio elaborou uma ficha técnica para cada experiência finalista, sendo os mesmos contatados para complementação de informações da experiência. Para seleção das três práticas premiadas, foram enviadas as fichas técnicas dos 11 finalistas a um time de jurados especiais, formado por oito comunicadores que tiveram a missão de julgar as experiencias finalistas sob a perspectiva do usuário. Cada membro deste júri votou individualmente e a soma dos votos trouxe o resultado das três experiências vencedoras.

O objetivo deste artigo é trazer a visão dos organizadores do Prêmio sobre os resultados obtidos, as lições aprendidas e reflexões sobre a experiência. Para isso, buscamos fazer uma análise quantitativa, qualitativa e transversal das experiências inscritas neste Prêmio, a luz da Política Nacional de Atenção Básica Brasileira e dos mandatos da Organização Pan-americana de Saúde / Organização Mundial de Saúde, considerando 
principalmente a Estratégia de Saúde Universal. ${ }^{1,2}$

\section{INTRODUÇÃO À ANÁLISE}

Desde o momento da sua concepção, o intuito do Prêmio APS Forte não foi apenas de identificar e reconhecer as melhores experiências de ampliação de acesso, mas também de captar e interpretar os elementos que estimulam as equipes técnicas da APS do SUS a inovar as práticas de atenção à saúde. Usando uma metáfora atual, tentamos olhar para a floresta e não apenas para as árvores. Trata-se de uma tarefa ambiciosa e complexa, porque as centenas de experiências apresentadas desenham um panorama muito heterogêneo, com uma exuberante diversidade de situações, problemas de saúde, contextos territoriais, marcos institucionais, tipologias de soluções e porte dos resultados alcançados. 0 dilema de um sistema único para um Brasil rico em diversidade.

Para realizar este exercício interpretativo, a análise das experiências foi conduzida de acordo a alguns parâmetros, como o perfil das equipes que narraram a própria prática, o território na qual foi desenvolvida, as características intrínsecas do desafio enfrentado e a sua coerência com as diretrizes da Política Nacional de Atenção Básica (PNAB). ${ }^{1}$

É importante destacar que este tipo de análise horizontal das experiências do Prêmio acarreta algumas limitações. Em primeiro lugar, um exercício deste tipo, qualitativo e interpretativo, implica num certo nível de subjetividade. A lente através da qual se olham os casos acaba influenciando a interpretação e a avaliação.

Outra limitação importante está relacionada com o fato que a avaliação das experiências está baseada apenas nas informações contidas nos textos sintéticos previstos pelos formulários padronizados, com o risco de limitar a capacidade de captar os elementos inovadores e os pontos de força das intervenções realizadas.

Apesar destas restrições, esta análise habilitou a geração de algumas hipóteses interpretativas, visando proporcionar aos responsáveis pela formulação de políticas elementos concretos e baseados na vivência daqueles que - em definitiva - são os protagonistas de qualquer processo profundo e sustentável de mudança, rumo ao fortalecimento da APS e do SUS. Acreditamos também que os resultados desta análise fornecem elementos para orientar pesquisas sobre os desafios contemporâneos da atenção primária.

\section{EM TODO O BRASIL, EQUIPES DE ESF MOTIVADAS E MUNICÍPIOS}

\section{COMPROMETIDOS COM A APS}

Ao analisar os dados relativos ao perfil das equipes participantes, fica claro o protagonismo das equipes de Saúde da Família (ESF) que, juntos com as equipes multiprofissionais dos Núcleos de Estratégia Saúde da Família, apresentaram mais da metade dos trabalhos apresentados ao prêmio.

O engajamento dos profissionais da ESF com o prêmio é alentador. São eles os protagonistas principais da APS e deles depende em grande medida a capacidade resolutiva e, em geral, o sucesso das práticas de saúde. A alta participação desta categoria reflete a sua motivação e compromisso com a atuação na APS, fator decisivo para o desempenho do sistema. 
Figura 1 - proporção dos perfis de participantes do Prêmio APS Forte.

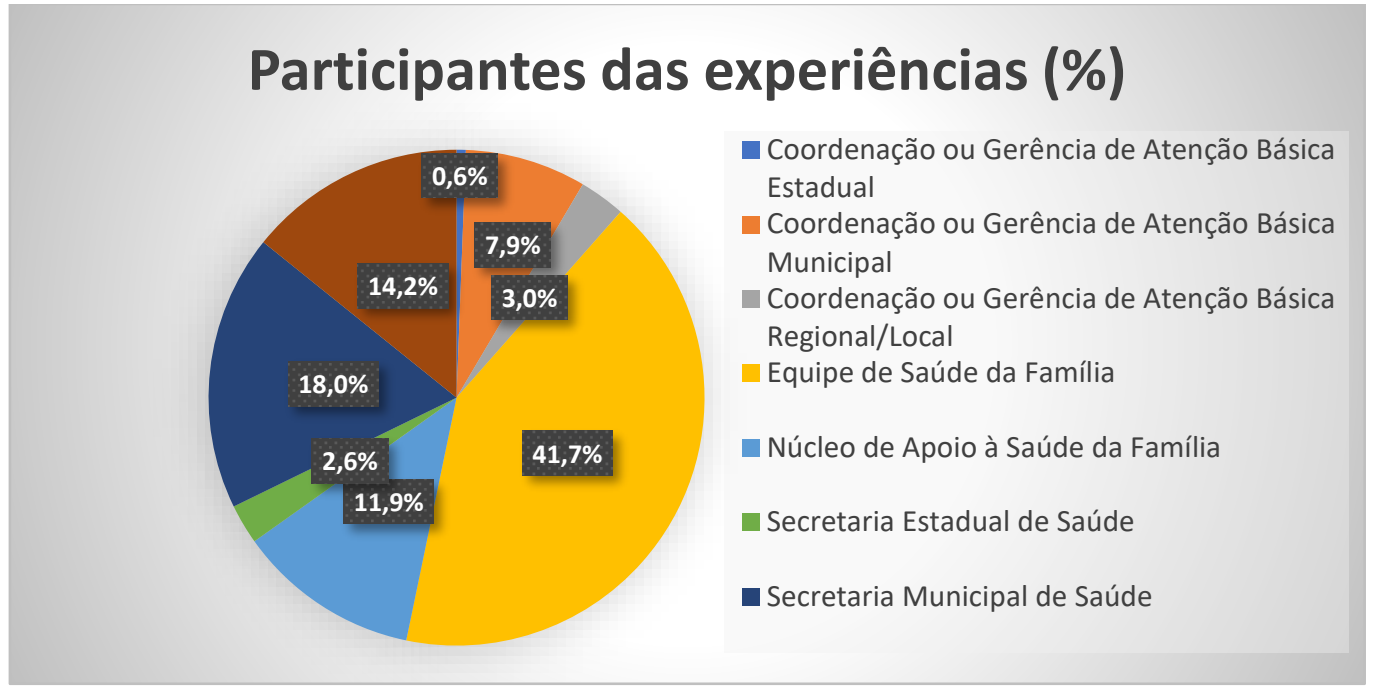

Fonte: Elaborado pelos autores.

Quando analisamos os profissionais envolvidos nas experiências apresentadas, temos alguns resultados interessantes. A tabela abaixo apresenta a frequência com que profissionais de saúde que trabalham na APS foram citados no campo descritivo "Como você desenvolveu a experiência?" do formulário de inscrição do Prêmio APS Forte:

Tabela 1 - Categorias profissionais com atuação na APS citadas no Desenvolvimento das experiências do Prêmio APS Forte.

\begin{tabular}{|l|c|}
\hline \multicolumn{1}{|c|}{ Categoria profissional } & $\begin{array}{c}\text { Número de experiências em que é } \\
\text { citado no "desenvolvimento" }\end{array}$ \\
\hline Enfermeira(o) & 294 \\
\hline Agente Comunitário de Saúde & 281 \\
\hline Médica(o) & 261 \\
\hline Dentista & 136 \\
\hline Psicóloga(o) & 110 \\
\hline Fisioterapeuta & 80 \\
\hline Auxiliar/Técnica(o) de Enfermagem & 79 \\
\hline Educador(a) físico & 57 \\
\hline Farmacêutica(o) & 56 \\
\hline Assistente Social & 54 \\
\hline Auxiliar/técnica(o) de Saúde Bucal & 44 \\
\hline Terapeuta Ocupacional & 15 \\
\hline Fonte: Elaboração dos autores. & \\
\hline
\end{tabular}

Fonte: Elaboração dos autores.

Embora tenhamos um modelo de assistência à saúde no geral bastante médico centrado, é interessante observar o protagonismo de outras categorias profissionais nos fazeres da APS. 0 
enfermeiro é o profissional mais citado nas experiências, indicativo do papel central que este desempenha em qualquer processo de cuidado dentro da APS. Logo após, o Agente Comunitário de Saúde, um profissional que foi fundamental no desenvolvimento da Estratégia Saúde da Família e vem sendo questionado sobre sua relevância e papel em tempos recentes. O conjunto de experiências que envolvem os ACS mostra que eles ainda têm muito a contribuir para a APS brasileira e estão envolvidos em processos fundamentais de territorialização, vínculo com a comunidade e educação em saúde. ${ }^{3}$

A representatividade das Secretarias Municipais de Saúde (SMS) também é uma boa notícia. Elas estão em uma posição de privilégio para transmitir o olhar sistêmico neste âmbito do SUS, além da sua absoluta relevância na construção de redes integradas de atenção. 0 bom desempenho na coordenação do cuidado, um dos atributos da APS, possibilita o desenvolvimento harmônico de redes de atenção e está estreitamente vinculado ao trabalho das secretarias municipais de saúde. Estas observações induzem a pensar que a alta participação das SMS signifique uma crescente consciência entre os gestores municipais do papel central da atenção primária na construção e coordenação das redes de atenção.

Outra variável que foi estudada é a procedência territorial das experiências. A Tabela 2 mostra os estados de origem das 1.239 experiências consideradas como elegíveis. Destacam em participação os estados de São Paulo (SP) com 201 experiências, Santa Catarina (SC) com 146 e Minas Gerais (MG) com 142. Em termos proporcionais, esses três estados juntos representam o 39,4\% dos trabalhos enviados ao prêmio.
Se tomamos como referência a cobertura populacional com ESF, a elevada participação de SC e MG é coerente com suas altas coberturas (90,6\% e $89,2 \%$, respectivamente). Por outro lado, a elevada participação do estado de SP no ranking deve-se ao expressivo número de equipes de APS implantadas, apesar de uma cobertura relativamente baixa $(41,2 \%$, a segunda menor a nível nacional). O estado que realmente destaca neste grupo dos três primeiros em envio de trabalhos é SC, que com 1.742 equipes implantadas, número expressivamente menor que nos casos de SP e MG, enviou ao prêmio 146 experiências, ocupando o segundo lugar entre os estados do Brasil.

Finalmente, com o intuito de corrigir a distorção introduzida pelas diferenças em tamanho populacional e número de equipes, calculamos um "coeficiente de participação" que relaciona o número de trabalhos enviados, com o total das equipes implantadas nos estados.

O uso deste coeficiente de participação leva à um ranking bastante diferente ao que responde apenas aos números absolutos. $O$ destaque mais interessante nesta perspectiva é para o DF, que apesar de uma cobertura relativamente baixa (32\%) teve o maior coeficiente de participação com um expressivo 51,9. Em segundo lugar, aparece Santa Catarina, o estado mais consistente em participação desde os diferentes ângulos de análise, totalizando um coeficiente de 38,6. Em terceiro lugar encontramos Amazonas (22,6\%), cujo ótimo resultado ficava ocultado no ranking obtido pelos números absolutos. 
Tabela 2 - Participação das equipes de saúde da família (ESF e NASF) no prêmio em relação com implantação nos estados

\begin{tabular}{|c|c|c|c|c|c|c|c|}
\hline UF & $\begin{array}{c}\text { ESF } \\
\text { implantadas }\end{array}$ & Cobertura ESF & $\begin{array}{c}\text { NASF } \\
\text { implantadas }\end{array}$ & $\begin{array}{c}\text { Total ESF e } \\
\text { NASF }\end{array}$ & $\begin{array}{l}\text { Experiências } \\
\text { inscritas } \\
\text { total }\end{array}$ & $\begin{array}{c}\text { Experiências } \\
\text { Inscritas ESFe } \\
\text { NASF }\end{array}$ & $\begin{array}{l}\text { Coef iciente de } \\
\text { participa ção } \\
\text { X } 1000\end{array}$ \\
\hline AC & 218 & $96,90 \% 6$ & 27 & 245 & 3 & 2 & 8,2 \\
\hline AL & 863 & 90,2096 & 145 & 1008 & 35 & 20 & 19,8 \\
\hline AM & 668 & 60,5096 & 84 & 752 & 33 & 17 & 22,6 \\
\hline AP & 133 & 62,4096 & 22 & 155 & 3 & 1 & 6,5 \\
\hline BA & 3546 & 81,3096 & 479 & 4025 & 54 & 32 & 8,0 \\
\hline$C E$ & 2463 & $96,80 \% 6$ & 313 & 2776 & 61 & 33 & 11.9 \\
\hline DF & 259 & 32,0056 & 30 & 289 & 27 & 15 & 51,9 \\
\hline ES & 717 & 64,4096 & 36 & 753 & 27 & 11 & 14,6 \\
\hline Go & 1392 & $74,60 \%$ & 242 & 1634 & 46 & 31 & 19,0 \\
\hline MA & 2049 & $100,00 \%$ & 266 & 2315 & 33 & 7 & 3,0 \\
\hline MG & 5322 & $89,20 \%$ & 962 & 6284 & 142 & 72 & 11,5 \\
\hline MS & 561 & 74,8096 & 77 & 638 & 12 & 10 & 15,7 \\
\hline MT & 710 & 77,0086 & 98 & 808 & 16 & 9 & 11,1 \\
\hline PA & 1448 & $62,40 \% 6$ & 175 & 1623 & 18 & 10 & 6,2 \\
\hline PB & 1396 & $100,00 \%$ & 288 & 1684 & 29 & 26 & 15,4 \\
\hline PE & 2298 & $86,10 \% 6$ & 286 & 2584 & 38 & 13 & 5,0 \\
\hline $\mathrm{PI}$ & 1301 & 100,0096 & 262 & 1563 & 42 & 25 & 16,0 \\
\hline PR & 2287 & $71,70 \% 6$ & 327 & 2614 & 75 & 30 & 11,5 \\
\hline RJ & 2869 & 60,5096 & 215 & 3084 & 75 & 47 & 15,2 \\
\hline RN & 1019 & 100,0096 & 183 & 1202 & 22 & 17 & 14,1 \\
\hline Ro & 366 & $73,10 \%$ & 24 & 390 & 8 & 4 & 10,2 \\
\hline $\mathrm{RR}$ & 116 & $82,00 \%$ & 16 & 132 & 1 & 0 & 0 \\
\hline RS & 2064 & $63,80 \%$ & 231 & 2295 & 80 & 41 & 17,9 \\
\hline $\mathrm{SC}$ & 1742 & 90,6096 & 307 & 2049 & 146 & 79 & 38,6 \\
\hline SE & 615 & $96,60 \% 6$ & 81 & 696 & 3 & 2 & 2,9 \\
\hline SP & 5212 & 41,2096 & 572 & 5784 & 201 & 108 & 18,7 \\
\hline To & 485 & $100,00 \%$ & 145 & 630 & 9 & 3 & 4,8 \\
\hline Total & 42119 & 72,3096 & 5893 & 48012 & 1239 & 665 & 13,9 \\
\hline
\end{tabular}

Fontes: Dados de implantação e cobertura: Ministério da Saúde; Dados de participação no prêmio: OPAS; Coeficiente de participação: ESF + NASF impantados/experiências inscritas ESF + NASF x 100 


\section{OS DESAFIOS DAS EQUIPES DE APS PARTICIPANTES}

A análise dos temas que foram objeto das experiências participantes nos proporcionou inúmeros elementos que ajudam a identificar os problemas percebidos como prioritários pelas equipes de APS do SUS. Devido à grande heterogeneidade de problemas e soluções apresentadas, as experiencias apresentadas foram classificadas por temas (ver Tabela 3). Esta operação obviamente tem limitações, como, por exemplo, nos casos que abarcam mais de um tema. Mesmo assim, achamos suficientemente precisa para identificar as principais tendências.

Tabela 3 - Temas que foram objetos das experiencias participantes do Prêmio APS Forte

\begin{tabular}{|c|c|c|}
\hline TEMAS & № & $\%$ \\
\hline \multicolumn{3}{|c|}{ Ações centradas na Comunidade } \\
\hline Práticas Integrativas e Complementares & 80 & $6,50 \%$ \\
\hline Alimentação e Nutrição & 41 & $3,30 \%$ \\
\hline Atividade Física e Academia da Saúde & 56 & $4,50 \%$ \\
\hline Promoção e prevenção da Saúde & 97 & $7,80 \%$ \\
\hline Populações vulneráveis & 83 & $6,70 \%$ \\
\hline \multicolumn{3}{|c|}{ Ações centradas nas pessoas } \\
\hline Adolescente & 25 & $2,00 \%$ \\
\hline DCNT & 50 & $4,00 \%$ \\
\hline Idosos & 28 & $2,30 \%$ \\
\hline Homem & 12 & $1,00 \%$ \\
\hline Mulher & 21 & $1,70 \%$ \\
\hline Materno-infantil & 72 & $5,80 \%$ \\
\hline Saúde Mental & 59 & $4,80 \%$ \\
\hline ISTs & 32 & $2,60 \%$ \\
\hline Saúde Bucal & 83 & $6,70 \%$ \\
\hline Imunização & 51 & $4,10 \%$ \\
\hline \multicolumn{3}{|c|}{ Ações Centradas na Gestão e Organização do Trabalho } \\
\hline Recursos Humanos & 20 & $1,60 \%$ \\
\hline Enfermagem & 12 & $1,00 \%$ \\
\hline Vigilância epidemiológica e arboviroses & 36 & $2,90 \%$ \\
\hline Processo de trabalho & 160 & $12,90 \%$ \\
\hline Ampliação e flexibilização de horários & 47 & $3,80 \%$ \\
\hline Flexibilização de agendas & 52 & $4,20 \%$ \\
\hline TIC & 84 & $6,80 \%$ \\
\hline Ampliação de cobertura da ESF & 25 & $2,00 \%$ \\
\hline Contratualização & 13 & $1,00 \%$ \\
\hline Total Geral & 1.239 & $100 \%$ \\
\hline
\end{tabular}

Fonte: Elaboração dos autores 
Em primeiro lugar, chama positivamente a atenção das equipes com os atributos "derivados" da APS (competência cultural, orientação familiar e comunitária). Foi observado um elevado número de intervenções destinadas a grupos vulneráveis, algumas de grande criatividade e eficácia. Duas das três experiencias vencedoras, Salgueiro (RJ) e Abaetetuba (PA), consistem em inciativas projetadas à comunidade

\section{Os desafios da implementação de ações em promoção de saúde no cenário escolar: relato de experiência de um} grupo de crianças no Salgueiro

A Equipe de Saúde da Família que atende a comunidade do Salgueiro, na Zona Norte da cidade do Rio de Janeiro, observou o alto número de encaminhamentos de alunos da escola local por alterações de comportamento no ambiente escolar, a maioria antecipando diagnóstico de Transtorno do Déficit de Atenção/Hiperatividade (TDAH). Surgiu aí, para a ESF, a necessidade de avaliar não só as crianças encaminhadas, mas também os contextos familiar e social em que estavam inseridas. No caso, a comunidade do Salgueiro, área de alta vulnerabilidade social.

Foi criado o "Grupo das Crianças", com encontros na própria escola, com duração média de 45 minutos, em que os profissionais da ESF conduzem o grupo a partir de metodologias e dinâmicas previamente definidas, a fim de facilitar atividades em que as crianças possam demonstrar aquisições e atrasos no desenvolvimento infantil. $O$ objetivo era oferecer um cuidado diferente do habitual.

Como resultado do trabalho, as crianças assistidas reconhecem no grupo um lugar privilegiado. Foi valorizada a aproximação entre família, escola e saúde. Foi pactuada a escuta e a atenção para com as crianças, tanto pela escola como pela família. A experiência da ESF Salgueiro diagnosticou a prevalência alta de violência contra crianças (maus tratos) que gera stress nas crianças, recomendando que os diagnósticos psiquiátricos devem ser cuidadosamente elaborados por equipes competentes para evitar o risco de consequência a medicalização e patologização da infância.

O diagnóstico abrangente sobre como a violência afeta a neurofisiologia das crianças foi um divisor de águas neste cuidado. O desenvolvimento de afeto entre os participantes dissolve o contexto violento, é terapêutico e gera maior empatia dos médicos para com a população.

\section{Abaetetuba - PA: Menina de laço de fita: a ternura como essência, a luta como princípio e o empoderamento como} estratégia para a cidadania.

Em um município com apenas 53\% de cobertura de Saúde da Família e com altos índices de mortalidade de mulheres em idade fértil, de gravidez na adolescência e de casos de sífilis, HIV e Hepatites Virais, os profissionais da Estratégia de Saúde da Família viram a necessidade de qualificação para melhorar a abordagem à saúde sexual e reprodutiva dos cerca de 156 mil moradores de Abaetetuba-PA, levando em conta suas diversidades e singularidades ao longo do ciclo da vida.

A experiência envolveu ações de saúde com adolescentes, adultos e idosos, abordando sexualidade, valorização e respeito; atendimento aos adolescentes por meio de oficinas de artes nas unidades; atividades focadas na melhoria da qualidade de vida; ações de mobilização em torno de temas como: diversidade sexual, bulling, homofobia, cidadania e cultura de paz; atendimento clinico para os casos necessários e oriundos das demandas da comunidade, com garantia de uma rede de atenção instituída e em parceria com a Assistência Social, Conselho de Saúde e todos os serviços de saúde do município.

Iniciado em março de 2018, o projeto apresentou resultados no mesmo ano, com a melhoria de alguns indicadores de saúde, destacando: ampliação em $100 \%$ dos testes rápidos de Hepatite B, Sífilis e HIV; ampliação em $92 \%$ dos atendimentos de saúde sexual e reprodutiva e ampliação em 10 vezes das coletas de PCCU, para prevenção do câncer de colo de útero.

Mas mais que melhorar o acesso da população às ações de assistência à saúde, a experiência representou o envolvimento da população na garantia do direito à saúde, a partir da descoberta de potencialidades dentro dos territórios e da valorização dos saberes populares, possibilitando um espaço para construção de práticas de saúde que reduzam iniquidades e valorizem a cidadania.

Ainda neste grupo de ações destinadas à comunidade, vale destacar o interessante número de experiências que oferecem atividades em grupo, ou que realizam atividades de promoção da saúde, alimentação saudáveis e atividade física. $O$ conjunto dos casos que fazem 
parte desta categoria é expressivo, chegando a quase um terço do total das experiências participantes. A qualidade destes trabalhos mostra o compromisso das equipes para ampliar o acesso às pessoas que vivem em áreas remotas ou sujeitas a outras formas de vulnerabilidade social, melhorando os serviços existentes ou criando intervenções novas, coerentes com a cultura e os costumes locais, capazes de concretizar o direito a saúde e agregar valor para a comunidade. Também demonstra a valorização do trabalho de saúde pública e de valorização do território, mostrando que estes atributos podem e devem caminhar lado a lado com as dimensões clínicas e de cuidado individual.

Outro grupo consistente de experiencias, aproximadamente $20 \%$ do total, se concentra em iniciativas visadas a melhorar a assistência de saúde às pessoas. Nesta categoria, observamos muitas experiencias focadas nas diferentes fases do curso de vida. Dentre desta categoria, o binômio materno-infantil é o tema que emerge de forma mais expressiva. Já em outros casos o foco da ação foi centrado no controle das pessoas com afeções frequentes e com elevada carga de doença, como as DST e as DCNT.

Finalmente, um terceiro grupo de temas fortemente presentes nas experiências participantes (um terço do total) diz respeito a gestão e organização do trabalho dos serviços e das equipes de APS. As intervenções mais frequentes deste grupo visam aprimorar os processos de trabalho da unidade de saúde, como a experiência vencedora de Jaraguá do Sul (SC).

Papel do protocolo da enfermagem no processo de acolhimento e primeira consulta para zerar as filas na atenção primária em saúde no município de Jaraguá do Sul (SC)

O fortalecimento do papel da Enfermagem na APS é um dos caminhos adotados internacionalmente para a sustentabilidade de sistemas de saúde universais e que, nos últimos anos, ganha prioridade no sistema brasileiro.

A atualização do Protocolo de Enfermagem, por secretarias de saúde com apoio dos conselhos regionais de Enfermagem, está ampliando o acesso dos usuários nas unidades de saúde da APS. Jaraguá do Sul, cidade com quase 175 mil habitantes em Santa Catarina, registrava em novembro de 2018 cerca de 15.500 consultas/mês reprimidas, somando todas das 25 unidades básicas de saúde da cidade, gerando insatisfação e reclamação dos usuários.

A partir da experiência de Florianópolis, a Secretaria de Saúde de Jaraguá do Sul elaborou o seu Protocolo de Enfermagem, gerando mais segurança para a atuação dos enfermeiros da rede municipal. A consulta de Enfermagem, com prescrição de exames e medicamentos essenciais para a população, bem como o processo de acolhimento nas unidades de saúde, foram incorporados na rotina de trabalho dos enfermeiros, a partir de novembro de 2018.

Em maio de 2019, a secretaria zerou a fila de primeira consulta para a APS. O processo de convencimento da população ao novo modelo de atendimento da APS, por meio de ampla divulgação do Protocolo de Enfermagem na imprensa e nas redes sociais, assim como o apoio político da Câmara de Vereadores e do Conselho Municipal de Saúde foram fundamentais para a implantação da experiência.

Ainda nesta categoria, observa-se que a necessidade de melhorar a gestão é outro estímulo importante para a inovação nos serviços de APS. Otimizar os recursos, ampliando horários dos serviços e flexibilizando as agendas, parece ser um objetivo perseguidos por muitos. Vale ressaltar, neste grupo, o discreto número de iniciativas centradas no uso das tecnologias da informação e comunicação, que objetivam facilitar os usuários (marcação de consultas, telemedicina) e melhorar a qualidade das informações entre os profissionais de saúde, numa lógica de rede (prontuário eletrônico).

\section{ANÁLISE DAS EXPERIÊNCIAS PARTICIPANTES À LUZ DA POLÍTICA NACIONAL dE ATENÇÃo BÁSICA (PNAB)}

A leitura horizontal das experiências, não pode prescindir da análise de coerência com a Política Nacional de Atenção Básica do Brasil (PNAB), 
cujos princípios e diretrizes representam a referência oficial para a organização e operação da APS no Brasil.

A diretriz de território da PNAB parece ser central em algumas experiências, que se debruçam na descrição do território e da população beneficiada, mostrando capacidade de adequar as práticas ao contexto. Um dos projetos finalistas, do município de Tefé (AM), simboliza a difusa sensibilidade das equipes com as necessidades do território e das pessoas às quais as ações da APS são destinadas. Vale também a pena mencionar o caso de Porto Alegre (RS), que descreve o uso de uma ferramenta de mapeamento para estruturar a atenção com base em território e população.

Produzindo inclusão da população ribeirinha pelas ações da unidade básica de saúde fluvial do Município de Tefé, Amazonas

Esta experiência mostra que é possível fornecer saúde de qualidade e cidadania para comunidades ribeirinhas e para os povos da floresta. Desenvolvida em Tefé, cidade à $540 \mathrm{~km}$ de Manaus (AM), onde as ruas são rios, os serviços da Atenção Primária à Saúde tiveram que se adaptar às condições do território. A solução encontrada foi a construção de uma embarcação (chamada Vila Ega), para ser a Unidade de Saúde da Família Fluvial (USFF) formada por uma equipe multiprofissional de saúde. A embarcação atende comunidades que não tem estruturas físicas de Unidades Básicas de Saúde (UBS) e apoia o trabalho de outras quatro equipes tradicionais de saúde ribeirinhas.

Desde junho de 2017 até maio de 2019, foram realizadas 14 viagens pela USFF Vila Ega onde a equipe trabalha com uma carteira ampliada de serviços, oferecendo consultas médicas, de enfermagem, odontológicas, com psicólogos, atendimentos de pré-natal e consultas puerperal, serviços de vigilância, busca ativa de doenças e agravos nãotransmissíveis, exames laboratoriais, entre outros, além de vacinação. Os resultados de quase um ano da experiência são aumento das coberturas vacinais, zero morte materna, acesso pela primeira vez às consultas odontológicas, aumento no número de exames citopatológicos do cólo de útero em mulheres entre 25 a 64 anos, entre outros.

\section{O uso de Tecnologia da Informação em Saúde na Tomada de Decisão em Porto Alegre - RS}

Desde 2013, Porto Alegre atua como município piloto na implementação do e-SUS AB, se mantendo parceiro do Ministério da Saúde na qualificação dessa ferramenta. Reconhecendo algumas limitações que a ferramenta e-SUS apresentou no processamento de dados e lançamento de relatórios, o município desenvolveu ferramentas, através de Business Intelligence (BI), para pesquisa de dados, relatórios de gestão, gráficos e dashboards.

$\mathrm{O}$ desenvolvimento do $\mathrm{BI}$ e-SUS tem se tornado uma importante ferramenta de trabalho, possibilitando o monitoramento de indicadores, acompanhamento de usuários, e o planejamento das ações com base na obtenção de informações provenientes da APS e que refletem a realidade local. A nível de gestão municipal, o BI e-SUS apontou a necessidade de revisão do Plano Municipal de Saúde e Programação Anual de Saúde, havendo a reformulação de indicadores, metas e ações, com vistas a ampliação do acesso. A lista de usuários vinculados a cada serviço, assim como o número de usuários diferentes que acessaram os serviços nos últimos três anos tem possibilitado inovação na forma de redimensionamento de recursos humanos e na definição de prioridade de lotação dos trabalhadores. A ferramenta desenvolvida permitiu o diagnóstico situacional e planejamento, subsidiando a tomada de decisão e desencadeando a revisão e melhoria dos processos de trabalho e portanto, revelando-se com potencial para auxiliar na formulação das Políticas Públicas de Saúde.

Como era de se esperar, a melhoria do acesso, tema central do Prêmio APS Forte, é a diretiva da PNAB mais frequentemente contemplada pelas experiencias participantes. Neste universo, observa-se uma interessante diversidade de soluções e contextos em que o acesso é abordado, com destaque para comunidades remotas e desfavorecidas de conglomerados urbanos. A experiência "Les
Femmes", é um ótimo exemplo disso. Por ser centrada na interação entre as equipes de saúde e um grupo populacional altamente vulnerável (imigrantes haitianas), mostra como um melhor acesso pode ser alcançado atuando diretamente no modus vivendi da comunidade, saindo das paredes da unidade e interagindo com sua cotidianidade. 


\section{Grupo Les Femmes: um relato de experiência}

Experiência sobre a iniciativa de uma Equipe de Saúde da Família do município de Canoas, região metropolitana de Porto Alegre, que percebeu um aumento significativo da população imigrante haitiana no seu território. Então foi criado o Grupo Les Femmes, com o propósito de encontros mensais, para mulheres haitianas, onde vários assuntos pertinentes à saúde da mulher eram abordados, com ajuda de tradutores apoiadores da própria comunidade. Alguns materiais educativos chegaram a ser traduzidos para o crioulo, língua nativa dessa nova população.

Para além dos temas específicos da saúde, o grupo proporcionou a troca de saberes e o conhecimento da cultura do povo haitiano, permitindo que essas mulheres fossem inseridas no serviço de saúde, com reflexos na comunidade. Muitas questões tratadas no grupo foram além da dimensão saúde, como violência doméstica e direitos de assistência social.

O trabalho teve grande relevância para as haitianas assistidas e para a equipe da unidade, uma vez que proporcionou, além da melhora na comunicação através dos assuntos abordados no grupo, a integração dessas mulheres à comunidade e o acesso aos serviços.

\section{Programa Corujão da Saúde de Doresópolis/MG}

Profissionais da Unidade Básica de Saúde (UBS) de Doresópolis, em Minas Gerais, cidade com 1.500 habitantes, se reorganizaram para melhorar a assistência à saúde. Moradores que antes não conseguiam acessar a UBS no horário comercial, por morar na área rural (110 famílias) ou por trabalhar em outra cidade, contam há mais de um ano com a UBS aberta todas as terças e quintas-feiras, das $7 \mathrm{~h}$ à $21 \mathrm{~h}$.

O horário estendido na UBS "Pedro da Costa Lopes" aumentou os atendimentos da equipe multiprofissional de saúde. O médico, por exemplo, atende 20 pacientes a mais do no horário convencional. Outro resultado positivo foi a melhoria na qualidade de vida dos usuários portadores de doenças crônicas com comorbidades, que antes eram atendidos individualmente. Com o Programa Corujão da Saúde, os pacientes recebem atendimentos em grupos nas noites das terças-feiras.

São encontros coletivos para tratar de lombalgia, aplicação de Prática Integrativas e Complementares (PICS), como reiki, auriculoterapia, ioga, quiropraxia, grupos de atividade física e de tabagismo

O Programa Corujão da Saúde também organiza encontro comunitários, como a Noite Forrozeira da Saúde, promovendo a cidadania e reforçando o sentimento de união entre os moradores da cidade.

\section{Implantação do laboratório de inovação as condições crônicas em Santo Antônio do Monte - MG}

Construir um modelo de atenção às condições crônicas de saúde, com foco em hipertensão e diabetes, que seja não só eficaz e sustentável, mas que funcione de forma integrada entre a atenção primária e a secundária. Este foi o desafio do Laboratório de Inovação em Condições Crônicas (LIACC) de Santo Antônio do Monte, município mineiro de 26 mil habitantes, a $160 \mathrm{~km}$ de Belo Horizonte.

O projeto teve como base o cadastramento familiar, a classificação de risco familiar e a estratificação de risco individual, além do cuidado longitudinal e integrado aos serviços especializados (prontuário eletrônico e segunda opinião clínica à distância), de acordo com o risco apresentado por cada paciente. A ideia foi buscar novas formas de atenção a condições crônicas, buscando não só o envolvimento do paciente e seus familiares, mas também da comunidade envolvente, com intervenções no território para criação de recursos comunitários para o controle das condições crônicas (como equipamentos públicos para a prática de atividade física) e também de um sistema de parcerias com instituições públicas e comunitárias.

Entre os resultados, o fortalecimento da Atenção Primária no município, com reconhecimento dos usuários, beneficiados com a melhoria do acesso aos serviços de saúde, com aumento significativo no número de consultas e do percentual de hipertensos e diabéticos que já haviam realizado a classificação de risco familiar e a estratificação de risco. Pesquisa finalizada em 2018 nos prontuários mostrou que em torno de $85 \%$ dos diabéticos e $95 \%$ dos hipertensos realizaram a classificação de risco familiar, Em 2015, 35\% e 28\%, respectivamente, apresentavam tal estratificação. Já em 2018 foram 48,9\% e 55,2\%, nas duas respectivas condições analisadas. 
Outros casos escolhem fortalecer o acesso mediante estratégias diferentes, baseadas no uso de ferramentas gerenciais e na reorganização da atenção, como por exemplo a USF de Doresópolis, (MG), que amplia e flexibiliza o horário.

A diretriz da PNAB relativa ao vínculo e responsabilização entre as equipes e a população adscrita também está contemplado por muitas experiências participantes, que aparentam um vínculo próximo e fluido entre as equipes de APS, os indivíduos e as comunidades. Neste grupo, destacamos o caso de Santo Antônio do Monte (MG), sobre controle de pessoas com condições crônicas.

Inclusão social, interculturalidade, comunidades fragilizadas, interação entre as equipes e as unidades de APS com outros setores sociais como educação, trabalho, esportes e governos locais em geral, são temas presentes na maioria dos trabalhos, o que em nossa opinião guarda coerência com as diretrizes da PNAB e os atributos de orientação familiar e comunitária e competência cultural.

Com menor frequência, se observam intervenções objetivadas a melhorar a coordenação do cuidado, mostrando que a APS não é isenta aos problemas gerados pela dificuldade do sistema a se integrar em redes de atenção, uma das principais fragilidades do SUS. ${ }^{4}$

\section{AtençÃo PrimÁRIA À SAÚde No}

\section{BRASIL: LUZES E SOMBRAS DE UM RETRATO EM MOVIMENTO.}

Os cuidados individuais e a saúde coletiva são os dois pilares do modelo de atenção da APS. Porém, é frequente a narrativa que reduz à APS a um serviço de pobre qualidade, destinado a pessoas sem recursos. Esta concepção mutilada da APS deixa de lado importantes dimensões de política de saúde, que transcendem os cuidados preventivos e clínicos básicos. Felizmente, esta APS deformada não aparece nas experiências participantes, o que revela uma prática bem orientada e baseada nos valores e princípios de APS.
Em geral, a leitura das experiências confirma a boa saúde da APS no SUS. Apesar de restrições financeiras, de recursos, insumos e infraestrutura presentes na APS, os trabalhos enviados ao prêmio demostram compromisso, motivação e qualidade das equipes de APS. Observa-se um leque muito abrangente de iniciativas nascidas da realidade local, impulsionadas pela necessidade de melhorar o serviço, que desenvolvem soluções "pês no chão" e, na maioria das vezes, efetivas.

Desde a perspectiva das práticas de saúde, o perfil da APS que emerge das experiências participantes é um serviço que combina cuidados individuais com ações sobre grupos da comunidade, mediante intervenções sobre determinantes sociais da saúde. Este é o mundo natural da APS, que usa simultaneamente habilidades clínicas e de saúde coletiva, como foi muito bem destacado no texto de Sergio Minué, desta mesma revista.

Outra boa notícia. Embora as equipes de saúde da família sejam - de longe - os principais protagonistas, em alguns casos percebe-se que o sucesso da prática se deve a sua capacidade de trabalhar de forma coordenada com o sistema municipal. Esses casos - apesar de não serem numerosos - reforçam e potencializam o papel da APS como coordenadora do cuidado e qualificam, ainda que indiretamente, o sistema de saúde local.

Um denominador comum das experiências apresentadas pode ser considerado o foco na vulnerabilidade da população alvo. Pessoas de origem rural, ribeirinha ou de alguma forma remota, mas que mesmo vivendo em centros populacionais das regiões mais desenvolvidas e com bons indicadores sociais e econômicos, estão privadas de acesso à atenção básica de saúde. Alguns casos mostram a capacidade das equipes de interpretar as informações sobre o território sob a sua responsabilidade, o que habilita intervenções centradas na realidade local e baseadas nas necessidades das pessoas.

As experiências participantes mostram uma expressiva capacidade de inovar, independentemente do contexto e dos recursos disponíveis, movidos apenas pela vontade de 
melhorar os serviços às famílias e às comunidades servidas. Esta capacidade se expressa introduzindo práticas inovadoras em diversas áreas: parcerias institucionais; participação expressiva de usuários, servidores e comunidade; introdução de temáticas contemporâneas (cultura da paz, saúde sexual, diversidade, etc.); foco em pessoas em estado de vulnerabilidade, com estratégias diferenciadas; promoção da humanização, equidade, proteção de direitos humanos e sociais; qualificação das equipes e informação aos usuários; abordagem biopsicossocial dos problemas de saúde; utilização da tecnologia de informação e comunicação; uso das evidências nas práticas clínicas.

\section{CONSIDERAÇÕES FINAIS}

Em primeiro lugar, cabe destacar que as experiências participantes do Prêmio APS Forte são uma prova concreta que a narrativa de um servidor público indolente e passivo é totalmente falsa. As equipes de APS trabalham intensamente para garantir o acesso de todas e todos a uma atenção à saúde integral e de qualidade. O que motiva os esforços e, em alguns casos, os sacrifícios destas pessoas é a genuína identificação com a missão do SUS, de um sistema público universal, baseado no direito à saúde.

Apesar da louvável motivação e, em geral, dos bons resultados alcançados pelas experiências participantes, algumas dificuldades foram identificadas e analisadas. Estes obstáculos, em parte vinculados a problemas que afetam a totalidade do SUS, limitam o seu potencial e podem atrasar o seu movimento rumo à uma APS Forte.

Em sua maioria, as experiências ficam restritas ao mundo das equipes e unidades da APS e suas práticas habituais, sem abordar intencionalmente as relações com outras unidades e, em geral, com a rede de serviços. A histórica dificuldade de integração dos serviços do SUS em redes de atenção limita a capacidade resolutiva e continuidade do cuidado da APS.

Outro problema percebido é a difusa dificuldade dos autores em relatar suas experiencias de forma exaustiva, mesmo dentro dos limites impostos pelo formulário de adesão. A causa destas limitações, nem sempre foi possível entender as motivações que induziram a equipe a escolher uma determinada solução, ou dispor de dados convincentes sobre os resultados. Em muitos casos, mais do que a análise das necessidades, é a atenção a um problema imediato que orienta o alvo da intervenção. Muito foco no processo, menos na estratégia ou na avaliação.

Generalizando, este achado sugere uma escassa capacidade de valorizar os pontos de força da iniciativa e uma insuficiente preocupação (ou competência) em medir objetivamente os efeitos das práticas implementadas. Isso é um problema, porque a dificuldade em explicitar plenamente o valor da própria prática faz que o conhecimento fique restrito na equipe que o desenvolveu, perdendo a chance de compartilhá-lo.

A análise dos resultados do Prêmio mostra também a importância deste tipo de iniciativa para os gestores do SUS, que ao estimularem iniciativas semelhantes estarão cultivando o hábito entre as equipes da APS para o registro dos trabalhos, contribuindo para a construção de conhecimento e divulgação de boas práticas em seus territórios. $\mathrm{Na}$ sociedade contemporânea, baseada no conhecimento, estas habilidades são cada dia mais necessárias, mesmo em profissões que tradicionalmente não as priorizem.

Entre os relatos presentes na maioria dos textos enviados percebe-se que o principal fator motivador das equipes é um genuíno desejo de melhorar os serviços, mesmo quando isso significa sair da própria zona de conforto. Produzir algo valioso para os usuários e para as comunidades é o que os impulsiona. Mais do que impessoais incentivos financeiros, a valorização e o envolvimento representam os principais instrumentos que motivam essas pessoas. Colocá-las em rede, criar espaços para dar voz aos seus dilemas e saberes, reconhecer suas conquistas e avanços são ações que os gestores do SUS devem constantemente promover. 
Concluímos com uma observação talvez óbvia, porém não dispensável: a criatividade e a motivação das equipes participantes representam um bem público precioso, com um potencial extraordinário para informar políticas de saúde destinadas ao sucesso. Ao tirar o olhar das árvores, o Prêmio APS Forte nos há desvelado uma floresta de milhares de mãos levantadas, de gente desejosa em crescer e melhorar o SUS, que espera ser ouvida, ouvir e aprender com os outros. Reconhecer os conhecimentos e os esforços destas pessoas, empoderá-las, proporcionando ferramentas para fortalecer seus talentos, habilidades e vocações é imprescindível para um SUS sustentável. 


\section{NOtAS E REFERÊNCIAS}

1.Brasil. Ministério da Saúde. Portaria no 2.436, de 21 de setembro de 2017. Aprova a Política Nacional de Atenção Básica, estabelecendo a revisão de diretrizes para a organização da Atenção Básica, no âmbito do Sistema Único de Saúde (SUS). Brasília, DF: Ministério da Saúde, 2020. [internet]. [acesso em 2020 fev 07]. Disponível em: http://www.brasilsus.com.br/index.php/legislacoes/gabinete-doministro/16247-portaria-n-2-436-de-21-de-setembro-de-2017.

2.OMS, Healthy systems for universal health coverage - a joint vision for healthy lives. Disponível em: https://www.uhc2030.org/fileadmin/uploads/uhc2030/Documents/About UHC2030/mgt arrangemts docs/UHC2030 Official documents/UHC2030 vision paper WEB2.pdf>

3.ALMEIDA, P. F.; GIOVANELLA, L. Avaliação em atenção básica à saúde no Brasil: mapeamento e análise das pesquisas realizadas e/ou financiadas pelo Ministério da Saúde entre os anos de 2000 e 2006. Cadernos de Saúde Pública, Rio de Janeiro, v. 24, n. 8, p. 1727-1742, 2008.

4.Organização Pan-Americana da Saúde. Relatório 30 anos de SUS. Que SUS para 2030? Brasília; 2018. Available from: https://apsredes.org/wp- 